\title{
GALOIS SECTIONS IN ABSOLUTE ANABELIAN GEOMETRY
}

\author{
SHINICHI MOCHIZUKI
}

\begin{abstract}
We show that isomorphisms between arithmetic fundamental groups of hyperbolic curves over $p$-adic local fields preserve the decomposition groups of all closed points (respectively, closed points arising from torsion points of the underlying elliptic curve), whenever the hyperbolic curves in question are isogenous to hyperbolic curves of genus zero defined over a number field (respectively, are once-punctured elliptic curves [which are not necessarily defined over a number field]). We also show that, under certain conditions, such isomorphisms preserve certain canonical "integral structures" at the cusps [i.e., points at infinity] of the hyperbolic curve.
\end{abstract}

\section{Introduction}

In this paper, we continue our study of the absolute anabelian geometry of hyperbolic curves over $p$-adic local fields [i.e., finite extensions of the field of $p$-adic numbers, for some prime number $p$ ], begun in [Mzk2], [Mzk3]. In [Mzk3, Theorem 2.4], it was shown, as a consequence of the main theorem of [Mzk1], that certain categories of finite étale correspondences associated to a hyperbolic curve $X_{K}$ over a $p$-adic local field $K$ may be recovered from the profinite group structure of the étale fundamental group $\Pi_{X_{K}}$ of $X_{K}$. In the present paper, we generalize this result to show [again as a consequence of the main theorem of [Mzk1]] that certain categories of arbitrary dominant [i.e., not necessarily finite étale] correspondences associated to $X_{K}$ may be recovered from the profinite group structure of $\Pi_{X_{K}}$ [cf. Theorem 2.3]. We then apply this result to study the extent to which the decomposition groups associated to closed points of $X_{K}$ may be recovered from the profinite group structure of $\Pi_{X_{K}}$ [cf. Corollaries 2.5, 2.6, 3.2]. One result that is representative of these techniques is the following special case of Corollary 3.2:

Received September 21, 2004.

2000 Mathematics Subject Classification: Primary 14H30; Secondary 14H25. 
Theorem A. Let $K$ be a finite extension of $\mathbb{Q}_{p} ; X_{K}$ a hyperbolic curve of genus zero over $K$ which is, in fact, defined over a number field. Write $\Pi_{X_{K}}$ for the étale fundamental group of $X_{K}$. Then any automorphism of the profinite group $\Pi_{X_{K}}$ preserves the decomposition groups $\subseteq \Pi_{X_{K}}$ associated to the closed points of $X_{K}$.

This result may be regarded as a sort of [very] weak version of the "Section Conjecture" [cf., e.g., [Mzk1], Section 19 for more on the "Section Conjecture"]. Finally, in Section 4, we show, in the notation of Theorem A, that various canonical auxiliary structures associated to the decomposition groups of cusps of $X_{K}$ are also preserved by arbitrary automorphisms of $\Pi_{X_{K}}$ [cf. Corollary 4.11].

Acknowledgements. I would like to thank Akio Tamagawa for various useful comments, especially concerning the statement of Theorem A; Corollaries 2.8, 3.2 and the proof of Lemma 4.6.

\section{$\S 0$. Notations and conventions}

\section{Numbers:}

If $p$ is a prime number, then we shall denote by $\mathbb{Q}_{p}$ the field of $p$-adic numbers, i.e., the completion of the field of rational numbers $\mathbb{Q}$ with respect to the $p$-adic valuation of $\mathbb{Q}$. We shall refer to a field which is isomorphic to a finite extension of $\mathbb{Q}_{p}$ for some $p$ as a local field. [In particular, in this paper, all "local fields" are nonarchimedean.] A number field is defined to be a finite extension of the field of rational numbers $\mathbb{Q}$.

\section{Topological Groups:}

Let $G$ be a Hausdorff topological group, and $H \subseteq G$ a closed subgroup. Let us write

$$
Z_{G}(H) \stackrel{\text { def }}{=}\{g \in G \mid g \cdot h=h \cdot g, \forall h \in H\}
$$

for the centralizer of $H$ in $G$;

$$
N_{G}(H) \stackrel{\text { def }}{=}\left\{g \in G \mid g \cdot H \cdot g^{-1}=H\right\}
$$

for the normalizer of $H$ in $G$; and

$$
C_{G}(H) \stackrel{\text { def }}{=}\left\{g \in G \mid\left(g \cdot H \cdot g^{-1}\right) \cap H \text { has finite index in } H, g \cdot H \cdot g^{-1}\right\}
$$


for the commensurator of $H$ in $G$. Note that: (i) $Z_{G}(H), N_{G}(H)$ and $C_{G}(H)$ are subgroups of $G$; (ii) we have inclusions

$$
H, Z_{G}(H) \subseteq N_{G}(H) \subseteq C_{G}(H)
$$

and (iii) $H$ is normal in $N_{G}(H)$. If $H=C_{G}(H)$, then we shall say that $H$ is commensurably terminal in $G$. Note that $Z_{G}(H), N_{G}(H)$ are always closed in $G$, while $C_{G}(H)$ is not necessarily closed in $G$.

If $G_{1}, G_{2}$ are Hausdorff topological groups, then an outer homomorphism $G_{1} \rightarrow G_{2}$ is defined to be an equivalence class of continuous homomorphisms $G_{1} \rightarrow G_{2}$, where two such homomorphisms are considered equivalent if they differ by composition with an inner automorphism of $G_{2}$.

\section{Categories:}

Let $\mathcal{C}$ be a category. We shall denote the collections of objects and arrows of $\mathcal{C}$ by

$$
\mathrm{Ob}(\mathcal{C}) ; \quad \operatorname{Arr}(\mathcal{C})
$$

respectively. If $A \in \operatorname{Ob}(\mathcal{C})$ is an object of $\mathcal{C}$, then we shall denote by

$$
\mathcal{C}_{A}
$$

the category whose objects are morphisms $B \rightarrow A$ of $\mathcal{C}$ and whose morphisms (from an object $B_{1} \rightarrow A$ to an object $B_{2} \rightarrow A$ ) are $A$-morphisms $B_{1} \rightarrow B_{2}$ in $\mathcal{C}$.

We shall refer to a natural transformation between functors [from one category to another] all of whose component morphisms are isomorphisms as an isomorphism between the functors in question. A functor $\phi: \mathcal{C}_{1} \rightarrow$ $\mathcal{C}_{2}$ between categories $\mathcal{C}_{1}, \mathcal{C}_{2}$ will be called rigid if $\phi$ has no nontrivial automorphisms. A category $\mathcal{C}$ will be called slim if the natural functor $\mathcal{C}_{A} \rightarrow \mathcal{C}$ is rigid, for every $A \in \mathrm{Ob}(\mathcal{C})$.

Given two arrows $f_{i}: A_{i} \rightarrow B_{i}$ (where $i=1,2$ ) in a category $\mathcal{C}$, we shall refer to a commutative diagram

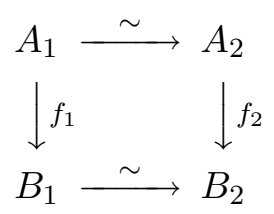

- where the horizontal arrows are isomorphisms in $\mathcal{C}$ - as an abstract equivalence from $f_{1}$ to $f_{2}$. If there exists an abstract equivalence from $f_{1}$ to $f_{2}$, then we shall say that $f_{1}, f_{2}$ are abstractly equivalent and write $f_{1} \stackrel{\text { abs }}{\approx} f_{2}$. 
Let $G$ be a profinite group. Then we recall that the category $\mathcal{B}(G)$ of finite sets with continuous $G$-action and morphisms of $G$-sets is slim if and only if $Z_{G}(H)=\{1\}$ for all open subgroups $H \subseteq G$.

\section{Curves:}

Suppose that $g \geq 0$ is an integer. Then if $S$ is a scheme, a family of curves of genus $g$

$$
X \longrightarrow S
$$

is defined to be a smooth, proper, geometrically connected morphism of schemes $X \rightarrow S$ whose geometric fibers are curves of genus $g$.

Suppose that $g, r \geq 0$ are integers such that $2 g-2+r>0$. We shall denote the moduli stack of $r$-pointed stable curves of genus $g$ (where we assume the points to be unordered) by $\overline{\mathcal{M}}_{g, r}[\mathrm{cf} .[\mathrm{DM}],[\mathrm{Knud}]$ for an exposition of the theory of such curves; strictly speaking, [Knud] treats the finite étale covering of $\overline{\mathcal{M}}_{g, r}$ determined by ordering the marked points]. The open substack $\mathcal{M}_{g, r} \subseteq \overline{\mathcal{M}}_{g, r}$ of smooth curves will be referred to as the moduli stack of smooth $r$-pointed stable curves of genus $g$ or, alternatively, as the moduli stack of hyperbolic curves of type $(g, r)$. The divisor at infinity $\overline{\mathcal{M}}_{g, r} \backslash \mathcal{M}_{g, r}$ of $\overline{\mathcal{M}}_{g, r}$ determines a log structure on $\overline{\mathcal{M}}_{g, r}$; denote the resulting $\log$ stack by $\overline{\mathcal{M}}_{g, r}^{\log }$.

A family of hyperbolic curves of type $(g, r)$

$$
X \longrightarrow S
$$

is defined to be a morphism which factors $X \hookrightarrow Y \rightarrow S$ as the composite of an open immersion $X \hookrightarrow Y$ onto the complement $Y \backslash D$ of a relative divisor $D \subseteq Y$ which is finite étale over $S$ of relative degree $r$, and a family $Y \rightarrow S$ of curves of genus $g$. One checks easily that, if $S$ is normal, then the pair $(Y, D)$ is unique up to canonical isomorphism. (Indeed, when $S$ is the spectrum of a field, this fact is well-known from the elementary theory of algebraic curves. Next, we consider an arbitrary connected normal $S$ on which a prime $l$ is invertible (which, by Zariski localization, we may assume without loss of generality). Denote by $S^{\prime} \rightarrow S$ the finite étale covering parametrizing orderings of the marked points and trivializations of the $l$ torsion points of the Jacobian of $Y$. Note that $S^{\prime} \rightarrow S$ is independent of the choice of $(Y, D)$, since (by the normality of $S), S^{\prime}$ may be constructed as the normalization of $S$ in the function field of $S^{\prime}$ (which is independent of the choice of $(Y, D)$ since the restriction of $(Y, D)$ to the generic point of $S$ has 
already been shown to be unique). Thus, the uniqueness of $(Y, D)$ follows by considering the classifying morphism (associated to $(Y, D)$ ) from $S^{\prime}$ to the finite étale covering of $\left(\mathcal{M}_{g, r}\right)_{\mathbb{Z}\left[\frac{1}{l}\right]}$ parametrizing orderings of the marked points and trivializations of the $l$-torsion points of the Jacobian [since this covering is well-known to be a scheme, for $l$ sufficiently large].) We shall refer to $Y$ (respectively, $D ; D ; D$ ) as the compactification (respectively, divisor at infinity; divisor of cusps; divisor of marked points) of $X$. A family of hyperbolic curves $X \rightarrow S$ is defined to be a morphism $X \rightarrow S$ such that the restriction of this morphism to each connected component of $S$ is a family of hyperbolic curves of type $(g, r)$ for some integers $(g, r)$ as above. If the divisor of cusps of a family of hyperbolic curves $X \rightarrow S$ forms a split finite étale covering over $S$, then we shall say that this family of hyperbolic curves is cuspidally split. A family of hyperbolic curves $X \rightarrow S$ of type $(0,3)$ (respectively, $(1,1)$ ) will be referred to as a tripod (respectively, once-punctured elliptic curve).

If $X_{K}$ (respectively, $Y_{L}$ ) is a hyperbolic curve over a field $K$ (respectively, $L)$, then we shall say that $X_{K}$ is isogenous to $Y_{L}$ if there exists a hyperbolic curve $Z_{M}$ over a field $M$ together with finite étale morphisms $Z_{M} \rightarrow X_{K}, Z_{M} \rightarrow Y_{L}$.

\section{§1. Brief review of anabelian geometry}

Let $K, L$ be local fields [cf. Section 0]; $X_{K}$ (respectively, $Y_{L}$ ) a hyperbolic curve [cf. Section 0] over $K$ (respectively, $L$ ). Any choice of basepoint for $X_{K}$ determines, up to inner automorphism, the étale fundamental group $\Pi_{X_{K}} \stackrel{\text { def }}{=} \pi_{1}\left(X_{K}\right)$ of $X_{K}$. Moreover, $\Pi_{X_{K}}$ fits into a natural exact sequence

$$
1 \longrightarrow \Delta_{X} \longrightarrow \Pi_{X_{K}} \longrightarrow G_{K} \longrightarrow 1
$$

where $G_{K}$ is the absolute Galois group of $K ; \Delta_{X}$, which is often referred to as the geometric fundamental group of $X_{K}$, is defined so as to make the sequence exact. Any choice of basepoint for $Y_{L}$ determines a similar exact sequence for $Y_{L}$.

Proposition 1.1. (First properties)

(i) $\Pi_{X_{K}}$ is slim $[$ cf. Section 0].

(ii) Every isomorphism of profinite groups $\alpha: \Pi_{X_{K}} \stackrel{\sim}{\rightarrow} \Pi_{Y_{L}}$ fits into a 
unique commutative diagram

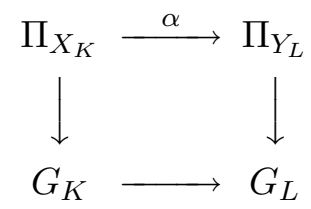

where the vertical arrows are the surjections of the natural exact sequence $(s)$ discussed above; the horizontal arrows are isomorphisms.

Proof. Assertion (i) (respectively, (ii)) follows from [Mzk2, Lemma 1.3.1] (respectively, [Mzk2, Lemma 1.3.8]).

TheOREM 1.2. (Anabelian theorem for hyperbolic curves over local fields) The étale fundamental group functor determines a bijection between the set of dominant morphisms of schemes

$$
X_{K} \longrightarrow Y_{L}
$$

and the set of open outer homomorphisms $\phi: \Pi_{X_{K}} \rightarrow \Pi_{Y_{L}}$ that fit into a commutative diagram

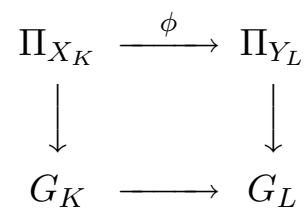

for which the induced morphism $G_{K} \rightarrow G_{L}$ is an open immersion [i.e., an isomorphism onto an open subgroup of $G_{L}$ ] which arises from an embedding of fields $L \hookrightarrow K$.

Proof. Recall that given a local field $M$, the topology of $M$ may be always be recovered solely from the field structure of $M$ by observing that the ring of integers $\mathcal{O}_{M}$ of $M$ is additively generated by $\mathcal{O}_{M}^{\times}$, and that $\mathcal{O}_{M}^{\times} \subseteq$ $M$ is equal to the subgroup of elements of $M^{\times}$that are infinitely divisible by powers of some prime number. In particular, the $\mathbb{Q}_{p}$-algebra structure of $M$ [for some suitable prime number $p$ ], as well as the prime number $p$ itself [i.e., the unique prime number $l$ such that $\mathcal{O}_{M}$ is not infinitely divisible by powers of $l$, may be recovered from the field structure of $M$. In a similar vein, given a function field in one variable $M^{\prime}$ over $M$, consideration of the discrete valuations on $M^{\prime}$ with trivial restriction to $M$ reveals that the subfield $M \subseteq M^{\prime}$ may be recovered — solely from the field structure of $M^{\prime}$ - as 
the subfield generated by the elements of $\left(M^{\prime}\right)^{\times}$that are infinitely divisible by powers of some prime number. In light of these remarks, Theorem 1.2 follows formally from [Mzk1, Theorem A].

Next, let us write $X_{K} \hookrightarrow \bar{X}_{K}$ for the compactification [cf. Section 0] of $X_{K}$. Let

$$
x \in \bar{X}_{K}
$$

be a closed point. Thus, $x$ determines, up to conjugation by an element of $\Pi_{X_{K}}$, a decomposition group:

$$
D_{x} \subseteq \Pi_{X_{K}}
$$

We shall refer to a closed subgroup of $\Pi_{X_{K}}$ which arises in this way as a decomposition group of $\Pi_{X_{K}}$. If $x$ is a cusp, then we shall refer to the decomposition group $D_{x}$ as cuspidal. Note that $D_{x}$ always surjects onto an open subgroup of $G_{K}$. Moreover, the subgroup

$$
I_{x} \stackrel{\text { def }}{=} D_{x} \cap \Delta_{X}
$$

is isomorphic to $\widehat{\mathbb{Z}}(1)$ [i.e., the profinite completion of $\mathbb{Z}$, Tate twisted once] (respectively, $\{1\}$ ) if $x$ is (respectively, is not) a cusp. We shall refer to a closed subgroup of $\Pi_{X_{K}}$ which is equal to " $I_{x}$ " for some cusp $x$ as a cuspidal geometric decomposition group.

THEOREM 1.3. (Decomposition groups)

(i) (Determination of the point) The closed point $x$ is completely determined by the conjugacy class of the closed subgroup $D_{x} \subseteq \Pi_{X_{K}}$. If $x$ is a cusp, then $x$ is completely determined by the conjugacy class of the closed subgroup $I_{x} \subseteq \Pi_{X_{K}}$.

(ii) (Commensurable terminality) The subgroup $D_{x}$ is commensurably terminal in $\Pi_{X_{K}}$. If $x$ is a cusp, then $D_{x}=C_{\Pi_{X_{K}}}(H)$ for any open subgroup $H \subseteq I_{x}$.

(iii) (Absoluteness of cuspidal decomposition groups) Every isomorphism of profinite groups

$$
\alpha: \Pi_{X_{K}} \stackrel{\sim}{\longrightarrow} \Pi_{Y_{L}}
$$

preserves cuspidal decomposition groups and cuspidal geometric decomposition groups.

(iv) (Cuspidal and noncuspidal decomposition groups) No noncuspidal decomposition group of $\Pi_{X_{K}}$ is contained in a cuspidal decomposition group of $\Pi_{X_{K}}$. 
Proof. The first half of assertion (i) follows, for instance, formally from [Mzk1, Theorem C]; the second half of assertion (i) follows from elementary facts about fundamental groups of topological surfaces. Assertion (ii) follows formally from assertion (i) and the definition of a "decomposition group". Assertion (iii) follows from assertion (ii) and [Mzk2, Lemma 1.3.9]. As for assertion (iv), we may assume, by passing to a finite étale covering of $X_{K}$, that $X_{K}$ is of genus $\geq 2$, so that $\bar{X}_{K}$ is still hyperbolic. Then assertion (iv) follows from assertion (i).

\section{$\S 2$. Categories of dominant morphisms}

Let $X_{K}$ be a hyperbolic curve over a field $K$. Write $X_{K} \hookrightarrow \bar{X}_{K}$ for the compactification of $X_{K}$.

DEFINITION 2.1.

(i) We shall refer to an open immersion

$$
X_{K} \longleftrightarrow Y_{K}
$$

as a partial compactification, or $P C$, for short, of $X_{K}$ if the natural open immersion $X_{K} \hookrightarrow \bar{X}_{K}$ factors as the composite of the given morphism $X_{K} \hookrightarrow Y_{K}$ with some open immersion $Y_{K} \hookrightarrow \bar{X}_{K}$. By abuse of notation, we shall also often speak of " $Y_{K}$ " as a PC of $X_{K}$.

(ii) If $X_{K} \hookrightarrow Y_{K}$ is a $\mathrm{PC}$ such that $Y_{K}$ is a hyperbolic curve, then we shall say that $X_{K} \hookrightarrow Y_{K}$ [or $Y_{K}$ ] is a hyperbolic partial compactification, or $H P C$, of $X_{K}$.

(iii) If $X_{K} \hookrightarrow Y_{K}$ is a PC such that the arrow "ฯ" is an isomorphism, then we shall say that $X_{K} \hookrightarrow Y_{K}$ [or $Y_{K}$ ] is a trivial partial compactification of $X_{K}$.

Now we define a "category of dominant localizations"

$$
\operatorname{DLoc}\left(X_{K}\right)
$$

associated to the hyperbolic curve $X_{K}$ as follows: The objects of this category are the hyperbolic partial compactifications

$$
Y \longleftrightarrow Z
$$

where $Y$ is a hyperbolic curve over some field [which is necessarily a finite separable extension of $K]$ that arises as a finite étale covering $Y \rightarrow X_{K}$. The 
morphisms of this category from an object $Y \hookrightarrow Z$ to an object $Y^{\prime} \hookrightarrow Z^{\prime}$ are diagrams of the form

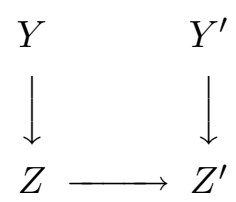

where the vertical morphisms are the given morphisms, and the horizontal morphism is a dominant morphism of schemes. By abuse of notation, we shall often simply refer to the horizontal arrow $Z \rightarrow Z^{\prime}$ as being a morphism of $\operatorname{DLoc}\left(X_{K}\right)$.

Similarly, by stipulating that all schemes appearing in the definition of the category $\operatorname{DLoc}\left(X_{K}\right)$ given in the preceding paragraph be equipped with $K$-structures [where we take the $K$-structure on $X_{K}$ to be the given $K$-structure] and that all morphisms be $K$-morphisms, we obtain a category

$$
\operatorname{DLoc}_{K}\left(X_{K}\right)
$$

together with a natural faithful functor $\operatorname{DLoc}_{K}\left(X_{K}\right) \rightarrow \operatorname{DLoc}\left(X_{K}\right)$.

Remark 2.2.0. Thus, the category $\operatorname{DLoc}\left(X_{K}\right)$ is reminiscent of the category "Loc $\left(X_{K}\right)$ " of [Mzk3, Section 2]. Indeed, there is a natural faithful functor

$$
\operatorname{Loc}\left(X_{K}\right) \longrightarrow \operatorname{DLoc}\left(X_{K}\right)
$$

whose essential image consists of the objects $Y \hookrightarrow Z$ which are trivial partial compactifications and the dominant morphisms $Z \rightarrow Z^{\prime}$ which are finite étale. In particular, if we denote by $\operatorname{Et}\left(X_{K}\right)$ the category of finite étale coverings of $X_{K}$ and morphisms over $X_{K}$, then we have natural faithful functors:

$$
\operatorname{Ét}\left(X_{K}\right) \longrightarrow \operatorname{Loc}\left(X_{K}\right) \longrightarrow \operatorname{DLoc}\left(X_{K}\right)
$$

Similarly, we have natural faithful functors: $\operatorname{Ét}\left(X_{K}\right) \rightarrow \operatorname{Loc}_{K}\left(X_{K}\right) \rightarrow$ $\operatorname{DLoc}_{K}\left(X_{K}\right)$.

Proposition 2.2. (Slimness of the category of dominant localizations) Suppose that $K$ is a local field. Then the categories $\operatorname{DLoc}\left(X_{K}\right), \operatorname{DLoc}_{K}\left(X_{K}\right)$ are slim.

Proof. Indeed, by using the various copies of "Ét $(Z)$ " [where, say, $Y \hookrightarrow Z$ is an object of $\left.\operatorname{DLoc}\left(X_{K}\right)\right]$ lying inside $\operatorname{DLoc}\left(X_{K}\right), \operatorname{DLoc}_{K}\left(X_{K}\right)$ [cf. Remark 2.2.0], the slimness of the categories $\operatorname{DLoc}\left(X_{K}\right), \operatorname{DLoc}_{K}\left(X_{K}\right)$ 
follows formally from Proposition 1.1, (i) [cf. also the discussion of slimness in Section 0].

Next, let us consider the category $\operatorname{DLoc}_{G_{K}}\left(\Pi_{X_{K}}\right)$ defined as follows: An object of this category is a surjection of profinite groups

$$
H \longrightarrow J
$$

where $H \subseteq \Pi_{X_{K}}$ is an open subgroup; $J$ is the quotient of $H$ by the closed normal subgroup generated by some collection of cuspidal geometric decomposition groups; and we assume that $J$ is "hyperbolic", in the sense that the image of $\Delta_{X} \cap H$ in $J$ is nonabelian. Given two objects $H_{i} \rightarrow J_{i}$, where $i=1,2$, of this category, a morphism in this category is defined to be a diagram of the form

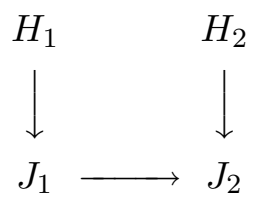

where the vertical morphisms are the given morphisms, and the horizontal morphism is an open outer homomorphism that is compatible with the various natural [open] outer homomorphisms from the $H_{i}, J_{i}$ to $G_{K}$.

Now we have the following analogue of [Mzk3, Theorem 2.4]:

ThEOREM 2.3. (Group-theoreticity of the category of dominant localizations) Let $K, L$ be local fields; $X_{K}$ (respectively, $Y_{L}$ ) a hyperbolic curve over $K$ (respectively, L). Then:

(i) The étale fundamental group functor determines equivalences of categories

$\operatorname{DLoc}_{K}\left(X_{K}\right) \stackrel{\sim}{\longrightarrow} \operatorname{DLoc}_{G_{K}}\left(\Pi_{X_{K}}\right) ; \quad \operatorname{DLoc}_{L}\left(Y_{L}\right) \stackrel{\sim}{\longrightarrow} \operatorname{DLoc}_{G_{L}}\left(\Pi_{Y_{L}}\right)$

(ii) Every isomorphism of profinite groups

$$
\alpha: \Pi_{X_{K}} \stackrel{\sim}{\longrightarrow} \Pi_{Y_{L}}
$$

induces an equivalence of categories

$$
\operatorname{DLoc}_{G_{K}}\left(\Pi_{X_{K}}\right) \stackrel{\sim}{\longrightarrow} \operatorname{DLoc}_{G_{L}}\left(\Pi_{Y_{L}}\right)
$$

hence also [by applying the equivalences of (i)] an equivalence of categories

$$
\operatorname{DLoc}_{K}\left(X_{K}\right) \stackrel{\sim}{\longrightarrow} \operatorname{DLoc}_{L}\left(Y_{L}\right)
$$


in a fashion that is functorial, up to unique isomorphisms of equivalences of categories, with respect to $\alpha$.

Proof. Indeed, assertion (i) follows formally from Theorem 1.2, while assertion (ii) follows, in light of Proposition 1.1, (ii); Theorem 1.3, (iii), formally from the definition of the categories "DLoc $G_{K}\left(\Pi_{X_{K}}\right)$ ", "DLoc $G_{L}\left(\Pi_{Y_{L}}\right)$ ". [Here, we note that the uniqueness of the isomorphisms of equivalences of categories involved follows from Proposition 2.2.]

Next, let

$$
D_{x} \subseteq \Pi_{X_{K}}
$$

be a decomposition group associated to some closed point $x \in \bar{X}_{K}$.

Definition 2.4. We shall say that $x$ or $D_{x}$ is of DLoc-type if $D_{x}$ admits an open subgroup that arises as the image via a morphism $Z \rightarrow X_{K}$ of $\operatorname{DLoc}_{K}\left(X_{K}\right)$ of some cuspidal decomposition group of $\Pi_{Z}$.

Corollary 2.5. (Group-theoreticity of decomposition groups of DLoctype) In the notation of Theorem 2.3, the isomorphism

$$
\alpha: \Pi_{X_{K}} \stackrel{\sim}{\longrightarrow} \Pi_{Y_{L}}
$$

preserves the decomposition groups of DLoc-type.

Proof. This follows immediately from the definitions; Theorem 2.3 [and its proof]; Theorem 1.3, (ii), (iii).

Corollary 2.6. (The case of once-punctured elliptic curves) In the notation of Theorem 2.3, let us suppose further that $X_{K}, Y_{L}$ are oncepunctured elliptic curves. Then the isomorphism

$$
\alpha: \Pi_{X_{K}} \stackrel{\sim}{\longrightarrow} \Pi_{Y_{L}}
$$

preserves the decomposition groups of the "torsion closed points" - i.e., the closed points that arise from torsion points of the underlying elliptic curve. Moreover, the resulting bijection between torsion closed points of $X_{K}$, $Y_{L}$ is compatible with the isomorphism on abelianizations of geometric fundamental groups $\Delta_{X}^{\mathrm{ab}} \stackrel{\sim}{\rightarrow} \Delta_{Y}^{\mathrm{ab}}$ - i.e., "Tate modules" — induced by $\alpha$. 
Proof. Indeed, if $n \geq 1$ is an integer, write

$$
\phi: Z_{K} \longrightarrow X_{K}
$$

for the finite étale covering determined by "multiplication by $n$ ". Note that this covering may also be described more group-theoretically as the covering associated to the open subgroup $H \subseteq \Pi_{X_{K}}$ [which is easily verified to be unique, up to conjugation in $\Pi_{X_{K}}$ ] such that: (i) $H$ contains a cuspidal decomposition group of $\Pi_{X_{K}}$; (ii) $H \cap \Delta_{X}$ is equal to the inverse image in $\Delta_{X}$ of the subgroup $n \cdot \Delta_{X}^{\mathrm{ab}} \subseteq \Delta_{X}^{\mathrm{ab}}$.

Observe that $Z_{K}$ admits $X_{K}$ as an HPC, by "filling in" all of the cusps other than the "origin". Thus, we obtain an open immersion

$$
\psi: Z_{K} \longleftrightarrow X_{K}
$$

- i.e., an object of $\operatorname{DLoc}_{K}\left(X_{K}\right)$, which exhibits the closed points of $X_{K}$ that arise from $n$-torsion points of the underlying elliptic curve as closed points of DLoc-type type. Thus, by transporting $\phi, \psi$ via the equivalences of Theorem 2.3, (i), and applying Theorem 1.3, (ii), (iii) [as in the proof of Corollary 2.5], we conclude that $\alpha$ preserves the decomposition groups of the torsion closed points. Finally, the compatibility with the induced morphism on Tate modules follows by considering the automorphisms of $Z_{K}$ over [i.e., relative to $\phi$ ] $X_{K}$, after possibly enlarging $K$.

Definition 2.7. We shall say that a closed point $x \in \bar{X}_{K}$ is algebraic if, for some finite extension $L$ of $K$, some hyperbolic curve $Y_{F}$ over a number field $F \subseteq L$, and some $L$-isomorphism $X_{L} \stackrel{\sim}{\rightarrow} Y_{L}$ [where $X_{L} \stackrel{\text { def }}{=} X_{K} \times_{K} L$, $\left.Y_{L} \stackrel{\text { def }}{=} Y_{F} \times_{F} L\right], x$ lies under a closed point $x_{L} \in \bar{X}_{L}$ which maps to a closed point of $\bar{Y}_{F}$ under the composite $\bar{X}_{L} \stackrel{\sim}{\rightarrow} \bar{Y}_{L} \rightarrow \bar{Y}_{F}$.

Remark 2.7.1. One verifies immediately that if a closed point $x \in \bar{X}_{K}$ is algebraic, then given any $L^{\prime}$-isomorphism

$$
X_{L^{\prime}} \stackrel{\sim}{\longrightarrow} Y_{L^{\prime}}^{\prime}
$$

[where $X_{L^{\prime}} \stackrel{\text { def }}{=} X_{K} \times_{K} L^{\prime} ; Y_{L^{\prime}}^{\prime} \stackrel{\text { def }}{=} Y_{F^{\prime}}^{\prime} \times_{F^{\prime}} L^{\prime} ; L^{\prime}$ is a finite extension of $K ; Y_{F^{\prime}}^{\prime}$ is a hyperbolic curve over a number field $F^{\prime} \subseteq L^{\prime}$, it holds that any point $x_{L^{\prime}} \in \bar{X}_{L^{\prime}}$ lying over $x$ maps to a closed point of $\bar{Y}_{F^{\prime}}^{\prime}$ under the composite $\bar{X}_{L^{\prime}} \stackrel{\sim}{\rightarrow} \bar{Y}_{L^{\prime}}^{\prime} \rightarrow \bar{Y}_{F^{\prime}}^{\prime}$ 
Corollary 2.8. (The case of genus zero) In the notation of Theorem 2.3 , let us suppose further that $X_{K}$ (respectively, $Y_{L}$ ) is isogenous [cf. Section 0] to a hyperbolic curve of genus zero. Then the isomorphism

$$
\alpha: \Pi_{X_{K}} \stackrel{\sim}{\longrightarrow} \Pi_{Y_{L}}
$$

preserves the decomposition groups of the algebraic closed points. In particular, $X_{K}$ is defined over a number field [or, equivalently: $X_{K}$ has at least one algebraic point] if and only if $Y_{L}$ is.

Proof. By Theorem 1.3, (ii), and [the " $\operatorname{Loc}_{K}(-)$ portion" - already contained in [Mzk3, Theorem 2.4] — of] Theorem 2.3, (ii), one reduces immediately to the case where both $X_{K}$ and $Y_{L}$ are of genus zero. Also, by Theorem 1.3, (ii), we may always enlarge $K, L$ without loss of generality; in particular, we may assume that $X_{K}, Y_{L}$ are cuspidally split, so that both curves admit a [cuspidally split] tripod as an HPC. Then we argue as in the proof of Corollary 2.6: That is to say, given any algebraic $x \in \bar{X}_{K}$, we observe that [after possibly enlarging $K$ ] there exists, by the definition of "algebraic" and the famous main result of [Belyi], a "Belyi map"

$$
\beta: X_{K} \longrightarrow \bar{X}_{K}
$$

that maps $x$, as well as all of the cusps of $X_{K}$, to cusps of $\bar{X}_{K}$, and, moreover, is unramified over the open subscheme of $\bar{X}_{K}$ determined by the tripod that forms an HPC for $X_{K}$. In particular, $\beta$ is unramified over the open subscheme $X_{K} \subseteq \bar{X}_{K}$. Put another way, there exists an open immersion $\phi: Z_{K} \hookrightarrow X_{K}$ [i.e., an HPC] such that the composite $\beta \circ \phi$ factors through $X_{K} \subseteq \bar{X}_{K}$ in such a way that the resulting morphism $\beta_{Z}: Z_{K} \rightarrow X_{K}$ is finite étale. In particular, $\beta_{Z}$ exhibits $\phi$ as an object of $\operatorname{DLoc}_{K}\left(X_{K}\right)$, and so $\phi$ exhibits $x$ as a closed point of DLoc-type. Thus, by transporting $\phi, \beta_{Z}$ via the equivalences of Theorem 2.3, (i), and applying Theorem 1.3, (ii), (iii) [as in the proof of Corollary 2.5], we conclude that $\alpha$ preserves the decomposition groups of algebraic closed points, as desired.

Remark 2.8.1. In fact, tracing through the proofs of Corollaries 2.6, 2.8 shows that in these proofs, we did not actually need to use the full "Hom" version of Theorem 1.2. That is to say, for these proofs, in fact the "isomorphism version" of Theorem 1.2 [i.e., the bijection between isomorphisms " $X_{K} \stackrel{\sim}{\rightarrow} Y_{L}$ " and certain isomorphisms " $\Pi_{X_{K}} \stackrel{\sim}{\rightarrow} \Pi_{Y_{L}}$ "], applied 
in combination with Theorem 1.3, (iii), is sufficient. Indeed, if we use the natural faithful functor discussed in Remark 2.2.0 to think of $\operatorname{Loc}_{K}\left(X_{K}\right)$ as a [not necessarily full!] subcategory of $\operatorname{DLoc}_{K}\left(X_{K}\right)$, then let us denote by

$$
\operatorname{Arr}\left(\operatorname{Loc}_{K}\left(X_{K}\right)\right) \subseteq \operatorname{OFLoc}_{K}\left(X_{K}\right) \subseteq \operatorname{Arr}\left(\operatorname{DLoc}_{K}\left(X_{K}\right)\right)
$$

the collection of arrows $Z \rightarrow Z^{\prime}$ of $\operatorname{DLoc}_{K}\left(X_{K}\right)$ which factor as the composite of an arrow $Z \rightarrow Z^{\prime \prime}\left[\right.$ of $\left.\operatorname{DLoc}_{K}\left(X_{K}\right)\right]$ which is an open immersion [i.e., an HPC] with an arrow $Z^{\prime \prime} \rightarrow Z^{\prime}\left[\right.$ of $\operatorname{DLoc}_{K}\left(X_{K}\right)$ ] which is finite étale. We shall refer to the arrows of $\operatorname{OFLoc}_{K}\left(X_{K}\right)$ as arrows of OF-type [i.e., "open immersion + finite étale" type]. Similarly, we define

$$
\operatorname{OFLoc}_{K}\left(\Pi_{X_{K}}\right) \subseteq \operatorname{Arr}\left(\operatorname{DLoc}_{G_{K}}\left(\Pi_{X_{K}}\right)\right)
$$

to be the collection of arrows $J_{1} \rightarrow J_{2}$ of $\operatorname{DLoc}_{G_{K}}\left(\Pi_{X_{K}}\right)$ that factor as the composite of a surjection $J_{1} \rightarrow J_{3}$ [in $\operatorname{DLoc}_{G_{K}}\left(\Pi_{X_{K}}\right)$ ] whose kernel is normally topologically generated by some collection of cuspidal geometric decomposition groups, with an open immersion $J_{3} \hookrightarrow J_{2}$ [in $\operatorname{DLoc}_{G_{K}}\left(\Pi_{X_{K}}\right)$ ]. Then [cf. Theorem 2.3, (ii), and its proof] we obtain an equivalence of categories

$$
\operatorname{DLoc}_{G_{K}}\left(\Pi_{X_{K}}\right) \stackrel{\sim}{\longrightarrow} \operatorname{DLoc}_{G_{L}}\left(\Pi_{Y_{L}}\right)
$$

whose induced map on "Arr(-)'s" maps $\operatorname{OFLoc}_{K}\left(\Pi_{X_{K}}\right)$ into OFLoc ${ }_{L}\left(\Pi_{Y_{L}}\right)$ by applying Proposition 1.1, (ii); Theorem 1.3, (iii) [i.e., without using Theorem 1.2 at all!]. Moreover, the isomorphism portion of Theorem 1.2 implies that the étale fundamental group functor induces a natural commutative diagram

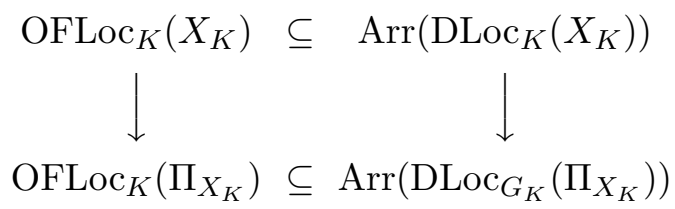

such that the vertical arrow on the left is "essentially surjective" - i.e., more precisely: induces a bijection on abstract equivalence [cf. Section 0] classes [defined relative to the category structures of $\operatorname{DLoc}_{K}\left(X_{K}\right)$, $\left.\operatorname{DLoc}_{G_{K}}\left(\Pi_{X_{K}}\right)\right]$ lying in $\operatorname{OFLoc}_{K}\left(X_{K}\right), \operatorname{OFLoc}_{K}\left(\Pi_{X_{K}}\right)$. Since the proofs of Corollaries 2.6, 2.8 only make use of arrows of OF-type, the bijection of abstract equivalence classes just observed, together with the equivalence $\operatorname{DLoc}_{G_{K}}\left(\Pi_{X_{K}}\right) \stackrel{\sim}{\rightarrow} \operatorname{DLoc}_{G_{L}}\left(\Pi_{Y_{L}}\right)$ - all of which involves only the isomorphism portion of Theorem 1.2 - are sufficient for the proofs of these categories, as claimed. 


\section{$\S 3$. Limits of Galois sections}

Let $X_{K}$ be a hyperbolic curve over a local field $K$. As in Sections 1, 2, we have an exact sequence:

$$
1 \longrightarrow \Delta_{X} \longrightarrow \Pi_{X_{K}} \longrightarrow G_{K} \longrightarrow 1
$$

Since $\Delta_{X}$ is topologically finitely generated, it follows that there exists a sequence of characteristic open subgroups

$$
\cdots \subseteq \Delta_{X}[j+1] \subseteq \Delta_{X}[j] \subseteq \cdots \subseteq \Delta_{X}
$$

[where $j$ ranges over the positive integers] of $\Delta_{X}$ such that $\bigcap_{j} \Delta_{X}[j]=\{1\}$. In particular, given any section

$$
\sigma: G_{K} \longrightarrow \Pi_{X_{K}}
$$

we obtain open subgroups

$$
\Pi_{X_{K}[j, \sigma]} \stackrel{\text { def }}{=} \operatorname{Im}(\sigma) \cdot \Delta_{X}[j] \subseteq \Pi_{X_{K}}
$$

[where $\operatorname{Im}(\sigma)$ denotes the image of $\sigma$ in $\Pi_{X_{K}}$ ] corresponding to a tower of finite étale coverings

$$
\cdots \longrightarrow X_{K}[j+1, \sigma] \longrightarrow X_{K}[j, \sigma] \longrightarrow \cdots \longrightarrow X_{K}
$$

of $X_{K}$ by hyperbolic curves over $K$.

The following lemma is reminiscent of the techniques of [Tama], [Mzk1]:

LEMmA 3.1. (Criterion for Galois sections associated to rational points) Suppose that $X_{K}$ is defined over a number field, i.e., there exists a hyperbolic curve $X_{K}$ over a number field $F \subseteq K$ such that $X_{K}=X_{F} \times_{F} K$. Let $\sigma: G_{K} \rightarrow \Pi_{X_{K}}$ be a section such that $\operatorname{Im}(\sigma)$ is not contained in any cuspidal decomposition group of $\Pi_{X_{K}}$. Then the following conditions on $\sigma$ are equivalent:

(i) $\sigma$ arises from a point $x \in X_{K}(K)$ [i.e., $\left." \operatorname{Im}(\sigma)=D_{x} "\right]$.

(ii) For every integer $j \geq 1, X_{K}[j, \sigma](K) \neq \emptyset$.

(iii) For every integer $j \geq 1, X_{K}[j, \sigma](K)^{\text {alg }} \neq \emptyset[$ where the superscript "alg" denotes the subset of algebraic $[K$-rational $]$ closed points $]$.

(iv) For every integer $j \geq 1, \Pi_{X_{K}[j, \sigma]}$ contains a decomposition group [i.e., relative to $\Pi_{X_{K}}$ ] of an algebraic closed point of $X_{K}$ that surjects onto $G_{K}$. 
Proof. (i) $\Longrightarrow$ (ii): It follows from the definitions that $x \in X_{K}(K)$ lifts to a point of $\in X_{K}[j, \sigma](K)$, for all $j \geq 1$, which implies (ii).

(iii) $\Longrightarrow$ (ii), (iv); (iv) $\Longrightarrow$ (iii): Immediate from the definitions.

(ii) $\Longrightarrow$ (i): For $j \geq 1$, choose points $x_{j} \in X_{K}[j, \sigma](K)$. Since the topological space

$$
\prod_{j \geq 1} \bar{X}_{K}[j, \sigma](K)
$$

is compact, it follows that there exists some infinite set of positive integers $J^{\prime}$ such that for any $j \geq 1$, the images of the $x_{j^{\prime}}$, where $j^{\prime} \geq j$, in

$$
\bar{X}_{K}[j, \sigma](K)
$$

converge to a point $y_{j} \in \bar{X}_{K}[j, \sigma](K)$. Moreover, note that, by the definition of $y_{j}$, it follows that if $j_{1}>j_{2}$, then $y_{j_{1}}$ maps to $y_{j_{2}}$ in $\bar{X}_{K}\left[j_{2}, \sigma\right](K)$. In particular, if we write $y \in \bar{X}_{K}(K)$ for the image of the $y_{j}$ in $\bar{X}_{K}(K)$, then it follows formally from the fact that the $y_{j}$ form a compatible sequence of points of the sets $\bar{X}_{K}[j, \sigma](K)$ that $\operatorname{Im}(\sigma)$ is contained in the decomposition group [well-defined up to conjugation] $D_{y}$. On the other hand, by our assumption that $\operatorname{Im}(\sigma)$ is not contained in any cuspidal decomposition group of $\Pi_{X_{K}}$, we conclude that $y$ is not a cusp, hence that " $\operatorname{Im}(\sigma)=D_{y}$ ", as desired.

(ii) $\Longrightarrow$ (iii): Given a point $x_{j} \in X_{K}[j, \sigma](K)$ with image $x \in X_{K}(K)=$ $X_{F}(K)$, it follows from "Krasner's lemma" [cf., e.g., [Kobl, pp. 69-70]] that one may approximate $x$ by a point $x^{\prime} \in X_{F}\left(F^{\prime}\right) \subseteq X_{F}(K)=X_{K}(K)$, where $F^{\prime} \subseteq K$ is a finite extension of $F$, which is sufficiently close to $x$ that [just like $x]$ it lifts to a point $x_{j}^{\prime} \in X_{K}[j, \sigma](K)$, which is necessarily algebraic, as desired.

Corollary 3.2. (Absoluteness of decomposition groups for genus zero) Let $K, L$ be local fields; $X_{K}$ (respectively, $Y_{L}$ ) a hyperbolic curve over $K$ (respectively, L), which is, in fact, defined over a number field. Suppose, moreover, that $X_{K}$ (respectively, $\left.Y_{L}\right)$ is isogenous [cf. Section 0] to a hyperbolic curve of genus zero. Then every isomorphism of profinite groups

$$
\alpha: \Pi_{X_{K}} \stackrel{\sim}{\longrightarrow} \Pi_{Y_{L}}
$$

preserves the decomposition groups of the closed points.

Proof. Indeed, Corollary 3.2 follows formally from Corollary 2.8; Theorem 1.3, (iii), (iv); and the equivalence (i) $\Longleftrightarrow$ (iv) of Lemma 3.1. 
Remark 3.2.1. Since any once-punctured elliptic curve is isogenous to a hyperbolic curve of genus zero, one might think, at first glance, that Corollary 2.6 is [essentially] a "special case" of Corollary 3.2. In fact, however, this is false, since Corollary 2.6 applies even to curves which are not necessarily defined over a number field.

\section{$\S 4$. Discrete and integral structures at cusps}

Let $X_{K}$ be a hyperbolic curve over a local field $K$; write $X_{K} \hookrightarrow \bar{X}_{K}$ for the compactification of $X_{K}$. Also, if $p$ is the residue characteristic of $K$, then we shall write $\widehat{\mathbb{Z}}^{\prime} \stackrel{\text { def }}{=} \widehat{\mathbb{Z}} / \mathbb{Z}_{p}$. Let

$$
D_{x} \subseteq \Pi_{X_{K}}
$$

be a decomposition group associated to some cusp $x \in \bar{X}_{K}(K)$. Then we have an exact sequence

$$
1 \longrightarrow I_{x}(\cong \widehat{\mathbb{Z}}(1)) \longrightarrow D_{x} \longrightarrow G_{K} \longrightarrow 1
$$

whose splittings form a torsor over

$$
H^{1}\left(G_{K}, \widehat{\mathbb{Z}}(1)\right) \cong\left(K^{\times}\right)^{\wedge}
$$

[where the " $\wedge$ " denotes the profinite completion]. If $\omega_{x}$ denotes the cotangent space to $\bar{X}_{K}$ at $x$, then any choice of a nonzero $\theta \in \omega_{x}$ determines a splitting of this torsor by considering the $\widehat{\mathbb{Z}}(1)$-torsor over the formal completion $\left(\bar{X}_{K}\right)_{x}$ [i.e., of $\bar{X}_{K}$ at $x$ ] given by taking $N$-th roots [as $N$ ranges over the positive integers] of any local coordinate $t \in \mathfrak{m}_{\bar{X}_{K}, x}$ such that $\left.d t\right|_{x}=\theta$. In particular, if the pointed stable curve associated to $X_{K}$ has stable reduction over $\mathcal{O}_{K}$, then the cotangent module to this stable reduction at the $\mathcal{O}_{K}$-valued point determined by $x$ determines a natural integral structure on $\omega_{x}$ [i.e., a rank one free $\mathcal{O}_{K}$-submodule of the one-dimensional $K$-vector space $\left.\omega_{x}\right]$. In particular, this integral structure determines a reduction of the structure group of the torsor of splittings considered above from $\left(K^{\times}\right)^{\wedge}$ to $\mathcal{O}_{K}^{\times}$.

\section{DEFINITION 4.1 .}

(i) If $\left(K^{\times}\right)^{\wedge} \rightarrow A$ is a continuous homomorphism of topological groups, then the torsor obtained from the torsor of splittings considered above by changing the structure group via this homomorphism will be referred to as the $A$-torsor at $x$. If, moreover, $B \subseteq A$ is a closed subgroup, then any 
reduction of the structure group of the $A$-torsor at $x$ from $A$ to $B$ will be referred to as a $B$-torsor structure at $x$.

(ii) $\mathrm{A} \mathcal{O}_{K^{-}}^{\times}\left(\right.$respectively, $\left.K^{\times}-\right)$torsor structure on the $\left(K^{\times}\right)^{\wedge}$-torsor at $x$ will be referred to as a(n) integral (respectively, discrete) structure on the cuspidal decomposition group $D_{x}$. Let us think of $\left(K^{\times}\right)^{\wedge} \otimes \widehat{\mathbb{Z}}^{\prime}$ as a quotient of $\left(K^{\times}\right)^{\wedge}$; write $\left(\mathcal{O}_{K}^{\times}\right)^{\prime},\left(K^{\times}\right)^{\prime}$ for the images of $\mathcal{O}_{K}^{\times}, K^{\times}$, respectively, in $\left(K^{\times}\right)^{\wedge} \otimes \widehat{\mathbb{Z}}^{\prime}$. Then a $\left(\mathcal{O}_{K}^{\times}\right)^{\prime}$ - (respectively, $\left.\left(K^{\times}\right)^{\prime}-\right)$ torsor structure on the $\left(K^{\times}\right)^{\wedge} \otimes \widehat{\mathbb{Z}}^{\prime}$-torsor at $x$ will be referred to as a(n) tame integral (respectively, tame discrete) structure on the cuspidal decomposition group $D_{x}$.

(iii) If $X_{K}$ has stable reduction over $\mathcal{O}_{K}$ (respectively, $X_{K}$ is arbitrary), then the particular integral (respectively, discrete) structure on $D_{x}$ arising [as discussed above] from a generator of the rank one free $\mathcal{O}_{K}$-submodule of $\omega_{x}$ determined by the stable reduction of $X_{K}$ (respectively, any nonzero element of $\omega_{x}$ ) will be referred to as the canonical integral (respectively, discrete) structure on the cuspidal decomposition group $D_{x}$. The canonical integral (respectively, discrete) structure on $D_{x}$ induces a tame integral (respectively, tame discrete) structure on $D_{x}$ which we shall also refer to as canonical.

(iv) An arbitrary closed point $x^{\prime}$ of $\bar{X}_{K}$ will be referred to as absolute if, for every $Y_{L}, \alpha$ as in Theorem 2.3, there exists a closed point $y^{\prime}$ of $\bar{Y}_{L}$ such that $\alpha\left(D_{x^{\prime}}\right)=D_{y^{\prime}}$. A nonconstant unit $U \in \Gamma\left(X_{K}, \mathcal{O}_{X_{K}}^{\times}\right)$on $X_{K}$ will be called coabsolute if $\bar{X}_{K}$ admits an absolute point at which $U$ is invertible. The hyperbolic curve $X_{K}$ will be called coabsolute if it admits a coabsolute unit. The hyperbolic curve $X_{K}$ will be called quasi-coabsolute if it is isogenous to a coabsolute hyperbolic curve. If $X_{K}$ has stable reduction over $\mathcal{O}_{K}$ (respectively, $X_{K}$ is arbitrary), then the cusp $x$ will be called integrally absolute (respectively, discretely absolute) if, for every $Y_{L}, \alpha$ as in Theorem 2.3, the isomorphism $D_{x} \stackrel{\sim}{\rightarrow} D_{y}$ [where $y$ is a cusp of $Y_{L}-\mathrm{cf}$. Theorem 1.3, (iii)] induced by $\alpha$ is compatible with the canonical integral (respectively, discrete) structures on $D_{x}, D_{y}$. Similarly, one has a notion of tamely integrally absolute and tamely discretely absolute cusps.

(v) The cusp $x$ will be called subprincipal if it is contained in the support of a cuspidal principal divisor on [i.e., principal divisor supported in the cusps of $] \bar{X}_{K}$. The hyperbolic curve $X_{K}$ will be called subprincipally ample if every cusp of $X_{K}$ is subprincipal. The hyperbolic curve $X_{K}$ will be called subprincipally quasi-ample if it is isogenous to a subprincipally ample hyperbolic curve. 
Remark 4.1.1. By Theorem 1.3, (iii), cusps are always absolute. By Corollaries 2.6, 3.2, once-punctured elliptic curves, as well as hyperbolic curves that are isogenous to a hyperbolic curve of genus zero which is defined over a number field, have infinitely many absolute points.

Next, let us write

$$
\mathcal{L} \stackrel{\text { def }}{=} \mathcal{O}_{\bar{X}_{K}}(x)
$$

for the line bundle determined by the cusp $x$;

$$
\mathbb{L} \longrightarrow \bar{X}_{K}
$$

for the geometric line bundle determined by $\mathcal{L}$; and

$$
(\mathbb{L} \supseteq) \mathbb{L}^{\times} \longrightarrow \bar{X}_{K}
$$

for the complement of the zero section in $\mathbb{L}$. Thus, the natural inclusion $\mathcal{O}_{\bar{X}_{K}} \hookrightarrow \mathcal{O}_{\bar{X}_{K}}(x)$ determines a section

$$
\bar{X}_{K} \longrightarrow \mathbb{L}
$$

whose restriction to $X_{K}$ determines a section $X_{K} \rightarrow \mathbb{L}^{\times}$, hence a morphism of fundamental groups:

$$
\Pi_{X_{K}} \longrightarrow \Pi_{\mathbb{L}^{\times}} \stackrel{\text { def }}{=} \pi_{1}\left(\mathbb{L}^{\times}\right)
$$

Lemma 4.2. (The line bundle associated to a cusp) Suppose that $X_{K}$ is of type $(g, r)$, where $g \geq 2, r=1$. Then:

(i) $\Pi_{\mathbb{L} \times}$ fits into a short exact sequence:

$$
1 \longrightarrow \widehat{\mathbb{Z}}(1) \longrightarrow \Pi_{\mathbb{L}^{\times}} \longrightarrow \Pi_{\bar{X}_{K}} \longrightarrow 1
$$

Moreover, the resulting extension class $\in H^{2}\left(\Pi_{\bar{X}_{K}}, \widehat{\mathbb{Z}}(1)\right)$ is the first Chern class of the line bundle $\mathcal{L}$.

(ii) The morphism of fundamental groups $\Pi_{X_{K}} \rightarrow \Pi_{\mathbb{L}^{\times}}$induces an isomorphism $I_{x} \stackrel{\sim}{\rightarrow} \operatorname{Ker}\left(\Pi_{\mathbb{L}^{\times}} \rightarrow \Pi_{\bar{X}_{K}}\right)$. In particular, the morphism $\Pi_{X_{K}} \rightarrow$ $\Pi_{\mathbb{L} \times}$ is surjective. by

(iii) Write $\Delta_{X / \bar{X}} \stackrel{\text { def }}{=} \operatorname{Ker}\left(\Pi_{X_{K}} \rightarrow \Pi_{\bar{X}_{K}}\right)$. Then the quotient of $\Delta_{X / \bar{X}}$

$$
\operatorname{Ker}\left(\Pi_{X_{K}} \rightarrow \Pi_{\mathbb{L}^{x}}\right) \subseteq \Delta_{X / \bar{X}}
$$

is the maximal quotient of $\Delta_{X / X}$ on which the conjugation action by $\Delta_{X}$ is trivial. 
Proof. Assertion (i) follows from [Mzk4, Lemmas 4.3, 4.4, 4.5]. Assertion (ii) is immediate from the discussion preceding Definition 4.1 involving roots of local coordinates. As for assertion (iii), write $Q_{1} \stackrel{\text { def }}{=}$ $\Delta_{X / \bar{X}} / \operatorname{Ker}\left(\Pi_{X_{K}} \rightarrow \Pi_{\mathbb{L}^{\times}}\right) ; Q_{2}$ for the maximal quotient of $\Delta_{X / \bar{X}}$ on which the conjugation action by $\Delta_{X}$ is trivial. Thus, we have a natural surjection $Q_{2} \rightarrow Q_{1}$. Now assertion (iii) follows from assertion (ii) and the well-known fact that $\Delta_{X / X}$ is topologically generated by the $\Delta_{X}$-conjugates of $I_{x}$.

Next, let us recall the notation of [Mzk2, Section 1.2]: By local class field theory, we have a natural isomorphism

$$
\left(K^{\times}\right)^{\wedge} \stackrel{\sim}{\longrightarrow} G_{K}^{\mathrm{ab}}
$$

which we may use to think of the group of roots of unity of $\left(K^{\times}\right)^{\wedge}$ as a subgroup:

$$
\boldsymbol{\mu}_{\mathbb{Q} / \mathbb{Z}}(K) \subseteq G_{K}^{\mathrm{ab}}
$$

Also, we recall [cf. [Mzk2, Proposition 1.2.1, (iv)]] that the subgroup $K^{\times} \subseteq$ $\left(K^{\times}\right)^{\wedge} \stackrel{\sim}{\rightarrow} G_{K}$ may be recovered group-theoretically from the profinite group structure of $G_{K}$. Allowing " $K$ " to vary among the various finite extensions of a given $K$ inside an algebraic closure $\bar{K}$ of $K$, we obtain groups:

$$
\boldsymbol{\mu}_{\mathbb{Q} / \mathbb{Z}}(\bar{K}) ; \quad \boldsymbol{\mu}_{\widehat{\mathbb{Z}}}(\bar{K}) \stackrel{\text { def }}{=} \operatorname{Hom}\left(\mathbb{Q} / \mathbb{Z}, \boldsymbol{\mu}_{\mathbb{Q} / \mathbb{Z}}(\bar{K})\right) ; \quad \boldsymbol{\mu}_{\widehat{\mathbb{Z}}^{\prime}}(\bar{K}) \stackrel{\text { def }}{=} \boldsymbol{\mu}_{\widehat{\mathbb{Z}}}(\bar{K}) \otimes \widehat{\mathbb{Z}}^{\prime}
$$

In particular, by considering roots of local coordinates as in the discussion preceding Definition 4 .1, we obtain a natural isomorphism $\boldsymbol{\mu}_{\widehat{\mathbb{Z}}}(\bar{K}) \stackrel{\sim}{\rightarrow} I_{x}$.

THEOREM 4.3. (Rigidity of cuspidal geometric decomposition groups) In the notation of Theorem 2.3, suppose that $\alpha$ induces isomorphisms

$$
I_{x} \stackrel{\sim}{\longrightarrow} I_{y} ; \quad \boldsymbol{\mu}_{\widehat{\mathbb{Z}}}(\bar{K}) \stackrel{\sim}{\longrightarrow} \boldsymbol{\mu}_{\widehat{\mathbb{Z}}}(\bar{L})
$$

where $x \in \bar{X}_{K}(K)$ (respectively, $\left.y \in \bar{Y}_{L}(L)\right)$ is a cusp. Then these isomorphisms are compatible with the natural isomorphisms $\boldsymbol{\mu}_{\widehat{\mathbb{Z}}}(\bar{K}) \stackrel{\sim}{\rightarrow} I_{x}$; $\boldsymbol{\mu}_{\widehat{\mathbb{Z}}}(\bar{L}) \stackrel{\sim}{\rightarrow} I_{y}$.

Proof. Indeed, by replacing $X_{K}, Y_{L}$ by finite étale coverings, one reduces immediately to the case where both curves are of genus $\geq 2$. By "filling in" [cf. Theorem 1.3, (iii)] all of the cusps other than those of interest [i.e., $x, y$ ], we may assume, moreover, that $X_{K}, Y_{L}$ satisfy the hypotheses of Lemma 4.2. Thus, by Lemma 4.2, we conclude that the morphism

$$
H^{2}\left(\Delta_{\bar{X}}, I_{x}\right) \stackrel{\sim}{\longrightarrow} H^{2}\left(\Delta_{\bar{Y}}, I_{y}\right)
$$


induced by $\alpha$ is compatible with the extension classes of Lemma 4.2. On the other hand, by [Mzk2, Lemma 2.5, (ii)], the morphism

$$
H^{2}\left(\Delta_{\bar{X}}, \boldsymbol{\mu}_{\widehat{\mathbb{Z}}}(\bar{K})\right) \stackrel{\sim}{\longrightarrow} H^{2}\left(\Delta_{\bar{Y}}, \boldsymbol{\mu}_{\widehat{\mathbb{Z}}}(\bar{L})\right)
$$

induced by $\alpha$ is compatible with the elements determined by the Chern class

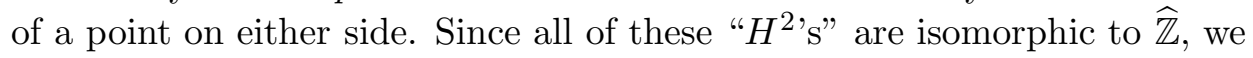
thus obtain the compatibility asserted in the statement of Theorem 4.3.

Proposition 4.4. (Tame integral absoluteness) Suppose that $X_{K}$ has stable reduction over $\mathcal{O}_{K}$. Then:

(i) Every cusp of $X_{K}$ is tamely integrally absolute.

(ii) A cusp of $X_{K}$ is discretely absolute if and only if it is integrally absolute.

Proof. Assertion (ii) follows formally from assertion (i) and the fact that the restriction of the projection $\widehat{\mathbb{Z}} \rightarrow \widehat{\mathbb{Z}}^{\prime}$ to $\mathbb{Z} \subseteq \widehat{\mathbb{Z}}$ is injective. Now we consider assertion (i). First, let us observe that it is immediate from the definitions that it suffices to prove assertion (i) after replacing $X_{K}$ by a finite étale covering of $X_{K}$ that extends to an admissible covering of the stable model of $X_{K}$. In particular, we may assume without loss of generality that every irreducible component of the normalization of the geometric special fiber of this stable model has genus $\geq 1$.

Next, let us recall the "étale quotient"

$$
\Pi_{X_{K}} \longrightarrow \Pi_{X_{K}}^{\mathrm{et}}
$$

of [Mzk2, Section 2]. Thus, the finite quotients of $\Pi_{X_{K}}^{\mathrm{et}}$ correspond to the coverings of $X_{K}$ that arise from finite étale coverings of the stable model of $X_{K}$ that are tamely ramified at the cusps. In particular, the quotient of $G_{K}$ determined by $\Pi_{X_{K}}^{\mathrm{et}}$ is the natural quotient $G_{K} \rightarrow G_{k}$, where $k$ is the residue field of $K$. If $x$ is a cusp of $X_{K}$, then [in light of our assumption that every irreducible component of the normalization of the geometric special fiber of the stable model has genus $\geq 1$ ] the quotient

$$
D_{x} \longrightarrow D_{x}^{\prime}
$$

determined by $\Pi_{X_{K}}^{\mathrm{et}}$ fits into an exact sequence:

$$
1 \longrightarrow I_{x}^{\prime} \longrightarrow D_{x}^{\prime} \longrightarrow G_{k} \longrightarrow 1
$$


[where $I_{x}^{\prime} \stackrel{\text { def }}{=} I_{x} \otimes \widehat{\mathbb{Z}}^{\prime}$. In particular, the splittings of this exact sequence form a torsor over $H^{1}\left(G_{k}, I_{x}^{\prime}\right) \cong k^{\times}$. These splittings may be thought of as elements of $H^{1}\left(D_{x}^{\prime}, I_{x}^{\prime}\right)$ whose restriction to $I_{x}^{\prime}$ is equal to the identity element of $H^{1}\left(I_{x}^{\prime}, I_{x}^{\prime}\right)=\operatorname{Hom}\left(I_{x}^{\prime}, I_{x}^{\prime}\right)$. Thus, unraveling the definitions, one verifies immediately that the pull-back to $D_{x}$ of any such element of $H^{1}\left(D_{x}^{\prime}, I_{x}^{\prime}\right)$ forms an element of $H^{1}\left(D_{x}, I_{x}^{\prime}\right)$ which determines the canonical tame integral structure on $D_{x}$. Since the étale quotient is compatible with isomorphisms $\alpha$ as in Theorem 2.3 [cf. [Mzk2, Lemma 2.2, (ii)]], we thus conclude that $x$ is tamely integrally absolute, as desired.

Proposition 4.5. (Absoluteness and coverings) Let $Z \rightarrow X_{K}$ be a finite étale covering. Let $z$ be a closed point of the compactification $\bar{Z}$ of $Z$ that maps to a closed point $x$ of $\bar{X}_{K}$. Then:

(i) $z$ is absolute (respectively, a discretely absolute cusp) if and only if $x$ is.

(ii) Suppose that $X_{K}, Z$ have stable reduction [over the rings of integers of their respective fields of constants]. Then $z$ is an integrally $\boldsymbol{a b}$ solute cusp if and only if $x$ is.

Proof. Assertion (i) is immediate from the definitions; [the "Loc $K(-)$ portion" — already contained in [Mzk3, Theorem 2.4] — of] Theorem 2.3, (ii) [cf. the proof of Corollary 2.8]; Theorem 1.3, (ii); the fact that $\widehat{\mathbb{Z}} / \mathbb{Z}$ is divisible. Assertion (ii) is immediate from assertion (i) and Proposition 4.4, (ii).

Before proceeding, we recall the following well-known result:

LEMmA 4.6. (Vanishing of Galois invariants of the Tate module) $W e$ have: $H^{0}\left(G_{K}, H^{1}\left(\Delta_{\bar{X}}, \boldsymbol{\mu}_{\widehat{\mathbb{Z}}}(\bar{K})\right)\right)=0$.

Proof. Since $T_{\bar{X}} \stackrel{\text { def }}{=} \Delta_{\bar{X}}^{\mathrm{ab}}$, i.e., the Tate module of the Jacobian $J_{X}$ of $\bar{X}_{K}$, is isomorphic to its Cartier dual, it suffices to show that $H^{0}\left(G_{K}, T_{\bar{X}}\right)=$ 0, i.e., that the torsion subgroup of $J_{X}(K)$ is finite. Since $J_{X}$ is a proper group scheme over $K$, it follows that the $p$-adic topology on $K$ determines a $p$-adic topology on $J_{X}(K)$ with respect to which $J_{X}(K)$ forms a compact p-adic Lie group. As is well-known [cf., e.g., [Serre, Chapter V, Section 7]], the exponential map for this $p$-adic Lie group determines an isomorphism of a certain open neighborhood of the identity of $J_{X}(K)$ with a free $\mathbb{Z}_{p^{-}}$ module of finite rank. Thus, the desired finiteness follows formally from this isomorphism, together with the compactness of $J_{X}(K)$. 
Remark 4.6.1. The author wishes to thank A. Tamagawa for informing him of the simple proof of Lemma 4.6 given above.

Next, let us observe that for any integer $N \geq 1$, the Kummer exact sequence

$$
1 \longrightarrow \boldsymbol{\mu}_{N} \longrightarrow \mathbb{G}_{\mathrm{m}} \longrightarrow \mathbb{G}_{\mathrm{m}} \longrightarrow 1
$$

[where $\mathbb{G}_{\mathrm{m}} \rightarrow \mathbb{G}_{\mathrm{m}}$ denotes the $N$-th power map on $\mathbb{G}_{\mathrm{m}} ; \boldsymbol{\mu}_{N}$ is defined so as to make the sequence exact] on the étale site of $X_{K}$ determines a long exact sequence in cohomology, hence, in particular, by letting $N$ vary, an injection

$$
H_{X} \stackrel{\text { def }}{=} \Gamma\left(X_{K}, \mathcal{O}_{X_{K}}^{\times}\right) \longleftrightarrow H_{X}^{\wedge} \hookrightarrow H^{1}\left(\Pi_{X_{K}}, \boldsymbol{\mu}_{\widehat{\mathbb{Z}}}(\bar{K})\right)
$$

[where we use the easily verified fact that $H_{X}$ is residually finite]. On the other hand, the Leray spectral sequence for the quotient $\Pi_{X_{K}} \rightarrow G_{K}$ yields an exact sequence:

$$
0 \longrightarrow\left(K^{\times}\right)^{\wedge} \longrightarrow H^{1}\left(\Pi_{X_{K}}, \boldsymbol{\mu}_{\widehat{\mathbb{Z}}}(\bar{K})\right) \longrightarrow D_{X} \stackrel{\text { def }}{=} H^{0}\left(G_{K}, H^{1}\left(\Delta_{X}, \boldsymbol{\mu}_{\widehat{\mathbb{Z}}}(\bar{K})\right)\right)
$$

Moreover, since, by Lemma $4.6, H^{0}\left(G_{K}, H^{1}\left(\Delta_{\bar{X}}, \boldsymbol{\mu}_{\widehat{\mathbb{Z}}}(\bar{K})\right)\right)=0$, it follows that, if we assume, for simplicity, that $X_{K}$ is cuspidally split, then restriction to the various " $I_{x}$ " in $\Delta_{X}$ determines [by applying the natural isomorphisms $\left.I_{x} \stackrel{\sim}{\rightarrow} \boldsymbol{\mu}_{\widehat{\mathbb{Z}}}(\bar{K})\right]$ an injection

$$
D_{X} \hookrightarrow P_{X} \stackrel{\text { def }}{=} \prod_{x} \widehat{\mathbb{Z}}
$$

[where the product ranges over the cusps $x$ of $X_{K}$ ]. In particular, we obtain exact sequences:

$$
0 \longrightarrow K^{\times} \longrightarrow H_{X} \longrightarrow P_{X} ; \quad 0 \longrightarrow\left(K^{\times}\right)^{\wedge} \longrightarrow H_{X}^{\wedge} \longrightarrow P_{X}
$$

Write $E_{X} \stackrel{\text { def }}{=} \operatorname{Im}\left(H_{X}\right) \subseteq P_{X}$ for the image of $H_{X}$ in $P_{X}$ [so we obtain an induced injection $\left.E_{X}^{\wedge} \hookrightarrow P_{X}\right]$. Thus, the maps

$$
H_{X} \longrightarrow P_{X} ; \quad H_{X}^{\wedge} \longrightarrow P_{X}
$$

are the maps obtained by associating to a function in $H_{X}$ its divisor of zeroes and poles. Put another way, $E_{X} \subseteq P_{X}$ may be characterized as the submodule of cuspidal principal divisors. 
Proposition 4.7. (Principal cuspidal divisors) In the notation of Theorem 2.3, assume that $X_{K}, Y_{L}$ are cuspidally split. Then the isomorphism

$$
P_{X} \stackrel{\sim}{\longrightarrow} P_{Y}
$$

induced [cf. Theorem 1.3, (iii)] by $\alpha$ maps $E_{X}$ onto $E_{Y}$.

Remark 4.7.1. In the statement of Proposition 4.7, as well as in the discussion to follow, we shall use similar notation for the objects associated to $Y_{L}$ to the notation used for the various objects just defined for $X_{K}$.

Proof. Write $J_{X}$ (respectively, $A_{X}$ ) for the Jacobian (respectively, Albanese variety) of $X_{K}$. Thus, the natural map $\bar{X}_{K} \rightarrow A_{X}$ determines a surjection on fundamental groups $\Pi_{\bar{X}_{K}} \rightarrow \Pi_{A_{X}} \stackrel{\text { def }}{=} \pi_{1}\left(A_{X}\right)$ whose kernel is the kernel of $\Delta_{\bar{X}} \rightarrow \Delta_{\bar{X}}^{\mathrm{ab}}$. In particular, any pair of sections of $\Pi_{\bar{X}_{K}} \rightarrow G_{K}$ determines a pair of sections of $\Pi_{A_{X}} \rightarrow G_{K}$ whose difference determines an element of $H^{1}\left(G_{K}, \Delta_{\bar{X}}^{\mathrm{ab}}\right)$. Moreover, if these sections arise from points $\in \bar{X}_{K}(K)$, then the resulting element of $H^{1}\left(G_{K}, \Delta_{X}^{\mathrm{ab}}\right)$ completely determines the point of $J_{X}(K)$ given by forming the difference of these two points [cf., e.g., [Mzk1], the discussion preceding Definition 6.2; [BK, Example 3.11]]. More generally, given any divisor of cusps on $\bar{X}_{K}$ with $\mathbb{Z}_{\text {- }}$ coefficients of degree 0 , the divisor is principal if and only if the resulting element of $H^{1}\left(G_{K}, \Delta \frac{\mathrm{ab}}{X}\right)$ vanishes. Since the sections of $\Pi_{\bar{X}_{K}} \rightarrow G_{K}$ arising from cusps are preserved by $\alpha$ [cf. Theorem 1.3, (iii)], we thus conclude that the isomorphism $P_{X} \rightarrow P_{Y}$ induced by $\alpha$ maps $E_{X}$ onto $E_{Y}$, as desired.

Definition 4.8. We shall say that $X_{K}$ is unitwise absolute if, in the notation of Theorem 2.3, the isomorphism

$$
H^{1}\left(\Pi_{X_{K}}, \boldsymbol{\mu}_{\widehat{\mathbb{Z}}}(\bar{K})\right) \stackrel{\sim}{\longrightarrow} H^{1}\left(\Pi_{Y_{L}}, \boldsymbol{\mu}_{\widehat{\mathbb{Z}}}(\bar{L})\right)
$$

induced by $\alpha$ maps the image of $\Gamma\left(X_{K}, \mathcal{O}_{X_{K}}^{\times}\right)$via the Kummer map onto the image of $\Gamma\left(Y_{L}, \mathcal{O}_{Y_{L}}^{\times}\right)$via the Kummer map.

Corollary 4.9. (Divisor-theoretic properties) In the notation of Theorem 2.3, let $x, y$ (respectively, $A \in E_{X}, B \in E_{Y}$ ) be cusps (respectively, cuspidal principal divisors) of $X_{K}, Y_{L}$, respectively, that correspond via $\alpha$ [cf. Proposition 4.7]. Then:

(i) $x$ is subprincipal if and only if $y$ is.

(ii) $A$ is the divisor of a coabsolute unit if and only if $B$ is. 
(iii) $X_{K}$ is coabsolute (respectively, quasi-coabsolute) if and only if $Y_{L}$ is.

(iv) $X_{K}$ is subprincipally ample (respectively, subprincipally quasi-ample) if and only if $Y_{L}$ is.

Proof. In light of Proposition 4.7, all of these statements follow formally from the definitions. Also, we note that for the various "quasi-" properties, one must apply [the "Loc $K(-)$ portion" - already contained in [Mzk3, Theorem 2.4] — of] Theorem 2.3, (ii), as in the proof of Corollary 2.8 .

TheOREM 4.10. (Units and canonical integral structures) Let $X_{K}$ be a hyperbolic curve over a local field $K$. Then:

(i) If $X_{K}$ is quasi-coabsolute, then it admits a discretely absolute cusp.

(ii) If $X_{K}$ admits a discretely absolute cusp or an absolute noncusp [i.e., an absolute point which is not a cusp], then $X_{K}$ is unitwise absolute.

(iii) If $X_{K}$ is unitwise absolute and subprincipally ample, then every cusp of $X_{K}$ is discretely absolute.

(iv) Suppose that $X_{K}$ has stable reduction over $\mathcal{O}_{K}$. Then if $X_{K}$ is quasi-coabsolute and subprincipally quasi-ample, then every cusp of $X_{K}$ is integrally absolute.

Proof. First, we consider assertion (i). In light of Proposition 4.5, (i), we may assume that $X_{K}$ is coabsolute. Let $U \in H_{X}$ be a coabsolute unit of $X_{K}$; let $x$ be a cusp of $X_{K}$ at which $U$ fails to be invertible. If $U$ has a zero of order $(\mathbb{Z} \ni) n \neq 0$ at $x$, then the restriction of the class

$$
\eta_{U} \in H^{1}\left(\Pi_{X_{K}}, \boldsymbol{\mu}_{\widehat{\mathbb{Z}}}(\bar{K})\right)
$$

determined by $U$ to $D_{x}$ determines a splitting of the torsor obtained by applying a change of structure group to the $\left(K^{\times}\right)^{\wedge}$-torsor at $x$ via the map $\left(K^{\times}\right)^{\wedge} \rightarrow\left(K^{\times}\right)^{\wedge}$ given by multiplication by $n$. Since $n \neq 0$, and $\widehat{\mathbb{Z}} / \mathbb{Z}$ is divisible, it thus follows that this splitting is sufficient to determine the canonical discrete structure on $D_{x}$. Let us write $\epsilon_{U} \in E_{X}$ for the image of $\eta_{U}$ in $E_{X}$. Then $\epsilon_{U}$ determines the set $\left(K^{\times}\right)^{\wedge} \cdot \eta_{U}$. On the other hand, since $U$ is coabsolute, it follows that $\bar{X}_{K}$ admits an absolute point $x^{\prime}$ at which $U$ is invertible. Thus, the subset

$$
K^{\times} \cdot \eta_{U} \subseteq\left(K^{\times}\right)^{\wedge} \cdot \eta_{U}
$$


may be characterized as the set of elements of $\left(K^{\times}\right)^{\wedge} \cdot \eta_{U}$ whose restriction to $D_{x^{\prime}}$ — which [by the invertibility of $U$ at $x^{\prime}$ ] necessarily lies in

$$
\left(K^{\times}\right)^{\wedge} \cong H^{1}\left(G_{K}, \boldsymbol{\mu}_{\widehat{\mathbb{Z}}}(\bar{K})\right) \subseteq H^{1}\left(D_{x^{\prime}}, \boldsymbol{\mu}_{\widehat{\mathbb{Z}}}(\bar{K})\right)
$$

— in fact lies inside $K^{\times} \subseteq\left(K^{\times}\right)^{\wedge}$. Thus, Theorem 4.3, Proposition 4.7, together with the absoluteness of $x^{\prime}$, imply that $x$ is discretely absolute, as desired.

Next, we consider assertion (ii). Let $x$ be a discretely absolute cusp or an absolute noncusp of $X_{K}$. Then, as in the argument applied in the proof of assertion (i), the image of $H_{X}$ in $H^{1}\left(\Pi_{X_{K}}, \boldsymbol{\mu}_{\widehat{\mathbb{Z}}}(\bar{K})\right)$ may be characterized as the set of elements lying over elements of $E_{X}$ whose restriction to $D_{x}$ determines a class in $H^{1}\left(D_{x}, \boldsymbol{\mu}_{\widehat{\mathbb{Z}}}(\bar{K})\right)$ that lies in the submodule of this cohomology module generated by the elements that define splittings "compatible with the canonical discrete structure on $D_{x}$ " [where in the noncuspidal case, we take this compatibility to mean that the restriction to $D_{x}$ lies in $K^{\times} \subseteq\left(K^{\times}\right)^{\wedge}$, as in the proof of assertion (i)]. Thus, assertion (ii) follows from Theorem 4.3, Proposition 4.7, together with the discrete absoluteness [in the cuspidal case] or absoluteness [in the noncuspidal case] of $x$.

Next, we observe that assertion (iii) follows via the argument applied in the proof of assertion (i), since the hypothesis that $X_{K}$ is unitwise absolute and subprincipally ample implies that for every cusp $x$ of $X_{K}$, there exists a unit $U \in H_{X}$ that is not invertible at $x$ and whose class in $H^{1}\left(\Pi_{X_{K}}, \boldsymbol{\mu}_{\widehat{\mathbb{Z}}}(\bar{K})\right)$ is mapped [in the notation of Theorem 2.3] to a class in $H^{1}\left(\Pi_{Y_{L}}, \boldsymbol{\mu}_{\widehat{\mathbb{Z}}}(\bar{L})\right)$ that lies in the image of $H_{Y}$.

Finally, we observe that assertion (iv) is a formal consequence of Proposition 4.4, (ii); Proposition 4.5, (i); assertions (i), (ii), (iii).

Corollary 4.11. (The case of genus zero) Let $X_{K}$ be a hyperbolic curve over a local field $K$ which is isogenous to a hyperbolic curve of genus zero. Then $X_{K}$ is quasi-coabsolute, subprincipally quasiample, and unitwise absolute. In particular, if $X_{K}$ has stable reduction over $\mathcal{O}_{K}$, then every cusp of $X_{K}$ is integrally absolute.

Proof. In light of Theorem 4.10, it suffices to show that if $X_{K}$ is of genus zero and cuspidally split, then $X_{K}$ is coabsolute and subprincipally ample. But since cusps are always absolute [cf. Theorem 1.3, (iii)], these properties follow formally from the following two elementary facts: (a) every divisor of degree 0 on $\bar{X}_{K}$ is principal; (b) $X_{K}$ has at least 3 cusps. 
In certain situations, the $\mathcal{O}_{K}^{\times}$-torsor determined by the canonical integral structure on the cuspidal decomposition group $D_{x}$ admits an even "finer reduction of structure group", as follows:

Corollary 4.12. (The case of once-punctured elliptic curves) Let $X_{K}$ be a once-punctured elliptic curve over a local field $K$ of residue characteristic $\neq 2$. Suppose that $X_{K}$ has stable reduction over $\mathcal{O}_{K}$. Also, if $n \geq 1$ is an integer, we shall write $\boldsymbol{\mu}_{n}(K) \subseteq \mathcal{O}_{K}^{\times}$for the subgroup of $n$-th roots of unity. Then there exists a $\boldsymbol{\mu}_{12}(K)$-torsor structure at the unique cusp $x$ of $X_{K}$ which is compatible with the canonical integral structure arising from the stable model $\mathcal{X}^{\log }$ and, moreover, is preserved by arbitrary automorphisms of $\Pi_{X_{K}}$.

Proof. We may assume without loss of generality that all of the 2torsion points of the underlying elliptic curve of $X_{K}$ are defined over $K$. Write $Y_{K} \rightarrow X_{K}$ for the Galois covering of degree 4 determined by the "multiplication by 2" map on the underlying elliptic curve of $X_{K}$ [so $Y_{K}$ is hyperbolic of type $(1,4)]$ and $\mathcal{Y}^{\log }$ for the stable model over $\operatorname{Spec}\left(\mathcal{O}_{K}\right)^{\log }$ [where the $\log$ structure on $\operatorname{Spec}\left(\mathcal{O}_{K}\right)$ is that determined by the closed point] of the smooth $\log$ curve $\bar{Y}_{K}^{\log }$ determined by $Y_{K}$. Also, let us write $e_{1}, e_{2}, e_{3}, e_{4}$ for the four cusps of $Y_{K}$.

Let $\alpha: \Pi_{X_{K}} \stackrel{\sim}{\rightarrow} \Pi_{X_{K}}$ be an automorphism of $\Pi_{X_{K}}$. Note that, by Theorem 1.3, (ii), any $\boldsymbol{\mu}_{12}(K)$-torsor structure at $x$ is preserved by arbitrary inner automorphisms of $\Pi_{X_{K}}$. Thus, we may assume [by composing with a suitable inner automorphism that induces a suitable element of $\left.\operatorname{Gal}\left(Y_{K} / X_{K}\right)\right]$ that the natural action of $\alpha$ on the cusps of $Y_{K}$ [cf. Theorem 1.3 , (iii)] preserves $e_{1}$.

Next, let us observe that [by the well-known definition of the group law on an elliptic curve; the definition of $\left.Y_{K} \rightarrow X_{K}\right]$ the divisor $D \stackrel{\text { def }}{=} 2\left[e_{1}\right]-2\left[e_{2}\right]$ on $Y_{K}$ is principal. Thus, there exists a unique rational function $f$ on $Y_{K}$ whose divisor of zeroes and poles is $D$ and whose value at $e_{3}$ is 1 . Since $D$ has multiplicity 2 at $e_{1}$, it follows that $f$ determines a $\boldsymbol{\mu}_{2}(K)$-torsor structure at $e_{1}$, hence also at $x$. Write $\eta$ for the Kummer class [i.e., the image under the Kummer map] of $f$. In the following, we shall write Kummer classes additively.

Now, observe that, by Proposition 4.7; Theorem 1.3, (iii), if $\alpha$ fixes all four cusps of $Y_{K}$, then it follows that $\alpha$ preserves the class $\eta$, hence also the $\boldsymbol{\mu}_{2}(K)$-torsor structure at $x$ determined by $\eta$. 
Next, let us write $\Sigma$ for the group of permutations of the three cusps $e_{2}, e_{3}, e_{4}$ that arise from automorphisms $\beta \in \operatorname{Aut}\left(\Pi_{X_{K}}\right)$ that preserve $e_{1}$. Thus, the order $s$ of $\Sigma$ divides 6 . Let $\beta_{1}, \ldots, \beta_{s} \in \operatorname{Aut}\left(\Pi_{X_{K}}\right)$ be a collection of automorphisms that give rise to the elements of $\Sigma$. Set:

$$
\eta^{\prime} \stackrel{\text { def }}{=}(6 / s) \cdot \sum_{j=1}^{s} \eta^{\beta_{j}}
$$

Since $Y_{K}$ is unitwise absolute by Corollary 4.11, it follows that $\eta^{\prime}$ arises from a rational function $f^{\prime}$ on $Y_{K}$ which has a pole of order 12 at $e_{1}$. In particular, $\eta^{\prime}$ determines a $\boldsymbol{\mu}_{12}(K)$-torsor structure at $e_{1}$, hence also at $x$. Moreover, it follows formally from the preceding observation concerning automorphisms $\alpha$ that fix all four cusps of $Y_{K}$ that arbitrary $\alpha$ [i.e., that are only assumed to fix $\left.e_{1}\right]$ preserve the $\boldsymbol{\mu}_{12}(K)$-torsor structure determined by $\eta^{\prime}$. Finally, the fact that this $\boldsymbol{\mu}_{12}(K)$-torsor structure is compatible with the canonical integral structure follows from the easily verified fact that the rational function $f$ is generically invertible [in light of our assumption that the residue characteristic of $K$ is $\neq 2$ ] on the special fiber of $\mathcal{Y}$. This completes the proof.

Remark 4.12.1. The number "12" appearing in Corollary 4.12 is interesting in light of the well-known fact that the line bundle on the moduli stack of elliptic curves determined by the cotangent bundle at the origin of the tautological family of elliptic curves has order 12 in the Picard group of this moduli stack.

Remark 4.12.2. It seems natural to expect that a(n) [perhaps somewhat more complicated] analogue of Corollary 4.12 should hold for more general hyperbolic curves $X_{K}$. This topic, however, lies beyond the scope of this paper.

\section{REFERENCES}

[Belyi] G. V. Belyi, On Galois extensions of a maximal cyclotomic field, Math. USSRIzv., 14 (1980), 247-256.

[BK] S. Bloch and K. Kato, L-Functions and Tamagawa Numbers, The Grothendieck Festschrift, Volume I, Birkhäuser (1990), pp. 333-400.

[DM] P. Deligne and D. Mumford, The Irreducibility of the Moduli Space of Curves of Given Genus, IHES Publ. Math., 36 (1969), 75-109.

[Knud] F. F. Knudsen, The Projectivity of the Moduli Space of Stable Curves, II, Math. Scand., 52 (1983), 161-199. 
[Kobl] N. Koblitz, $p$-adic Numbers, $p$-adic Analysis, and Zeta Functions, Graduate Texts in Mathematics 58, Springer Verlag, 1977.

[Mzk1] S. Mochizuki, The Local Pro-p Anabelian Geometry of Curves, Invent. Math., 138 (1999), 319-423.

[Mzk2] S. Mochizuki, The Absolute Anabelian Geometry of Hyperbolic Curves, Galois Theory and Modular Forms, Kluwer Academic Publishers (2003), pp. 77-122.

[Mzk3] S. Mochizuki, The Absolute Anabelian Geometry of Canonical Curves, Kazuya Kato's fiftieth birthday, Doc. Math. 2003, Extra Vol., pp. 609-640.

[Mzk4] S. Mochizuki, Topics Surrounding the Anabelian Geometry of Hyperbolic Curves, Galois Groups and Fundamental Groups, Mathematical Sciences Research Institute Publications 41, Cambridge University Press (2003), pp. 119-165.

[Serre] J.-P. Serre, Lie Algebras and Lie Groups, Lecture Notes in Mathematics 1500, Springer Verlag, 1992.

[Tama] A. Tamagawa, The Grothendieck Conjecture for Affine Curves, Compositio Math., 109 (1997), 135-194.

Research Institute for Mathematical Sciences

Kyoto University

Kyoto, 606-8502

Japan

motizuki@kurims.kyoto-u.ac.jp 